\title{
Peut-on justifier la suppression des Départements français ?
}

Une revue de la littérature

\section{Olivier THOMAS}

\author{
Maître de Conférences \\ Université Montpellier 1 \\ olivier.thomas@univ-montp1.fr \\ Chercheur associé au LEREPS \\ Université Toulouse 1 Capitole \\ olivier.thomas@ut-capitole.fr
}

700 avenue du Pic Saint Loup 34090 Montpellier (France) 


\section{Introduction : Le contexte du débat actuel}

Au printemps 2014, le premier ministre français Manuel Vals a surpris son auditoire quand il reprit à son compte l'ambitieux objectif de suppression d'un échelon de l'administration territoriale française : celui des Départements. Avec la révision de la valeur locative des différents impôts locaux, c'est sans doute la mesure qui a fait couler le plus d'encre au cours des dernières décennies sans jamais aboutir pour autant à une quelconque évolution. Malgré l'effet de surprise lié à cette annonce, ce nouveau projet ministériel, d'abord prévu à longue échéance, puis considéré quelques jours plus tard comme prioritaire à court terme, est en effet tout sauf une nouveauté.

Dès la fin des années 1940 on a commencé à débattre sur la réduction du nombre de Départements (Hertzog 2012). Depuis les années 1990, le Département «est fréquemment désigné comme l'échelon de trop, que ce soit par la DATAR, le Commissariat Général au Plan, par des rapports d'experts ou par des documents internes au Parti Socialiste » (Le Lidec 2007 p. 115). De même, dans un passé plus récent, le gouvernement Fillon s'était initialement fixé, avant de faire marche arrière, le même objectif, en reprenant l'une des propositions (la n ${ }^{\circ}$ 260) du Rapport Attali : faire disparaître en dix ans l'échelon départemental (Commission pour la Libération de la Croissance Française 2008). On retrouvait le même objectif au travers de la proposition de loi relative à la simplification de la carte des territoires du 21 janvier 2009 (Assemblée Nationale, 2009a) qui prévoyait très explicitement la suppression des Conseils Généraux en 2017. De très nombreuses contributions abondèrent dans le même sens (Balladur 2009, Warsmann 20008, pour la Réforme des Collectivités Locales 2009). 
Pourquoi supprimer les Départements ? On leur reprochait de ne pas disposer, en dépit de l'importance de leurs budgets ${ }^{1}$, d'une taille critique suffisante pour mettre en œuvre des politiques de développement économique local. Ils seraient alors devenus inadaptés à l'environnement socio économique de la France du $\mathrm{XXI}^{\circ}$ siècle. Certains analysent même leur disparition comme la manifestation d'un darwinisme territorial inéluctable dont des signes avant coureurs se seraient déjà manifestés notamment dans l'agglomération lyonnaise (Pinson \& Galimberti 2013, Pasquier 2013), et qui ne demanderaient donc qu'à s'étendre dans un avenir plus ou moins proche.

Bien que l'exécutif ait commandé plusieurs rapports dont les conclusions ont toutes convergé vers l'impérieuse nécessité d'une refonte radicale de l'architecture de l'administration locale, il est assez déroutant de constater qu'il n'a ensuite pas osé prendre la décision d'une telle réforme frontale, freiné par sa peur d'un Parlement plus «(soucieux) de préserver les intérêts de carrière des élus que de veiller à ceux des contribuables » (Le Lidec 2009 p. 479). De fait, «un état de dissonance cognitive apparaît entre ce qui est jugé économiquement souhaitable - une réforme radicale abaissant les coûts - et le politiquement faisable» (ibid. p. 478).

Ainsi, la loi de Réforme des Collectivités Territoriales ${ }^{2}$ (RCT) s'est voulue plus nuancée, en ne parlant plus explicitement de disparition des Départements (De Briant 2010). La frilosité des propos du Premier Ministre de l'époque, François Fillon, fut à cet égard révélatrice, quand il souligna la nécessité de préserver les effectifs employés par les Conseils Généraux ou encore de ne pas pénaliser les Départements «par des logiques ministérielles qui

\footnotetext{
${ }^{1} 71$ Md€ pour les départements soit un peu moins du triple du budget des Régions (27.5 Md€) mais seulement la moitié du budget du bloc communal (Communes et Intercommunalités : $137.7 \mathrm{Md} €$ ) : Observatoire des Finances Locales (2013).

${ }^{2}$ Loi n ${ }^{\circ} 2010-1563$ du 16 décembre 2010 de Réforme des Collectivités Territoriales.
} 
privilégieraient de façon excessive les mutualisations au niveau régional ${ }^{3}{ }$. Dans le même ordre d'esprit, la communication officielle usa de tous les moyens pour donner des gages de la survie des Départements, quitte à opérer des réinterprétations bien choisies : « (Régions et Départements): il n'a jamais été question de supprimer l'une ou l'autre de ces deux Collectivités Territoriales ${ }^{4} \gg$.

La loi RCT opta alors pour une voie plus indirecte : celle de l'instauration du statut si controversé, et immédiatement abrogé par le gouvernement Ayrault, du conseiller territorial. Ainsi aurait été possible, en évitant les oppositions frontales (notamment des lobbies d'élus locaux et d'associations de Collectivités Territoriales) de favoriser la montée en puissance des niveaux jugés d'avenir (Intercommunalités et Régions) en marginalisant progressivement les niveaux hérités du passé (Communes et Départements) sans pour autant, officiellement, remettre en cause leur existence.

Le caractère pusillanime de la démarche gouvernementale est d'autant plus étonnant si on considère que le Gouvernement, exceptionnellement, n'aurait pas eu à gérer l'hostilité de certains syndicats. En effet, «la CFDT aurait préféré un positionnement plus clair en faveur de la disparition, à terme, des Conseils Généraux, les Départements devenant alors le niveau des administrations déconcentrées des Régions » (Roulleau 2009 p. 51). A trop évoquer ce qui est possible, on en oublie de «mettre l'accent sur ce qui est souhaitable dès lors qu'on sait déjà que cela est nécessaire »(ibid. p.66).

\footnotetext{
${ }^{3}$ Service de Presse du Premier Ministre, 2009, « La Réforme de l'Administration Territoriale de l'Etat : l'Etat en mouvement », discours du Premier Ministre à La Défense, 16 novembre 2009, p.5.

${ }^{4}$ Ministère de l'Intérieur, de l'Outre Mer, des Collectivités Territoriales et de l'Immigration, 2010, «La Réforme des Collectivités Territoriales, une nouvelle impulsion pour les territoires », p.4.
} 
Comment justifier cette volte face, et cette inertie, concernant un projet qui semblait pourtant fédérer au delà des barrières partisanes et s'inscrire dans une évolution historique actée (Sadran 2009) ? Comment justifier le fait que «la réforme territoriale est en France un processus permanent d'évitement de la réforme (au terme duquel) les velléités de transformation radicale s'achèvent toujours par l'écrasement des velléitaires » (Négrier 2012 p. 74) ? Le niveau départemental est-il vraiment un constituant inamovible et irréfragable de notre organisation territoriale? Pourquoi «le législateur d'aujourd'hui refuserait-il d'envisager ce que celui de la Révolution avait fait pour adapter les institutions à la France de l'époque : modifier l'agencement des pouvoirs ?»(Guillaume 2010 p. 16). Pourquoi la « prophylaxie départementaliste » (Négrier 2012 p. 81) est-elle si prégnante ?

Cette contribution a pour objectif d'apporter certains éléments de réponse à cette problématique en se basant sur une analyse de la littérature concernant les Départements en particulier, et les tentatives de réforme de l'architecture territoriale française de façon plus générale. Deux axes nous semblent devoir être développés. Si les Départements ont survécu (et vont encore survivre quelques temps) aux attaques de leurs pourfendeurs, ce serait d'une part car on donnerait du crédit à des assertions erronées dépeignant le chaos qui résulterait de leur disparition. Et ce serait d'autre part car on passerait au contraire sous silence les avantages que revêtirait leur suppression. Nous nous proposons de détailler successivement chacun de ces groupes d'arguments dans les deux parties suivantes. 


\section{1: La réfutation des arguments fallacieux justifiant le maintien des}

\section{Départements}

Cette première partie tentera de montrer que le refus de la suppression de l'échelon départemental fait fréquemment appel à des arguments aisément réfutables. Ainsi, nous commencerons par souligner la confusion qui consiste à penser que l'on supprimera les Départements alors qu'il est en fait question des Conseils Généraux ${ }^{5}$. Puis notre argumentation remettra en question une autre croyance: celle de la concomitance entre disparition (éventuelle) des Conseils Généraux et disparition de leurs missions sociales. Enfin, nous questionnerons le fait que l'échelon départemental tire sa légitimité et sa pertinence de son ancienneté.

\section{1: Une confusion savamment entretenue entre Départements et Conseils}

\section{Généraux}

Même si les reproches en matière d'archaïsme faits aux Départements sont fortement récurrents, dès lors qu'il s'agit de les supprimer, la tendance à l'amnésie prend systématiquement le dessus et les citoyens font alors l'apologie de leur indéfectible attachement vis à vis de leur Département (Roulleau 2009). On pourrait épiloguer sur la force relative de cet attachement qui pousse les générations les moins anciennes à ne plus évoquer oralement l'objet de leur affection qu'au travers d'un simple numéro : celui du rang auquel leur Département apparaît par ordre alphabétique ${ }^{6}$, notamment sur les plaques d'immatriculation des véhicules.

\footnotetext{
${ }^{5}$ Et nous recourrons également à cet usage effectué dans le langage commun en évoquant dans notre propos la suppression des « Départements » pour signifier celle des Conseils Généraux.

${ }^{6}$ Exception faite des quelques changements de noms survenus après la création initiale des départements.
} 
A ce sujet, « les débats autour de la suppression de la référence départementale sur les plaques d'immatriculation des véhicules ont permis de prendre la mesure de l'attachement des français à cet échelon ». Cette citation n'est pas empruntée à un média régionaliste de vulgarisation mais à un rapport sénatorial sur la réorganisation territoriale (Krattinger \& Gourault 2009a p.13-14). Il est révélateur de la confusion, faite par une partie importante de la population nationale, entre Département et Conseil Général. Quand on évoque habituellement la suppression des premiers, c'est en fait de la disparition ${ }^{7}$ des seconds dont on parle!

Il n'est pas possible d'entretenir, au sein de l'opinion publique, la confusion entre ces deux termes pour ensuite justifier la nécessaire survie des «Départements » sur la seule base de sondages ${ }^{8}$ montrant l'attachement des français à leur territoire départemental. On peut justifier tout et son contraire par la volonté du peuple, en fonction du contexte en présence. Les français voudraient donc sacraliser en 2014 la gestion publique décentralisée, alors que si on avait respecté leur opinion, suite à l'avis émis lors du référendum de 1969, les Régions n'auraient jamais vu le jour.

Le Département n'est en fait qu'un zonage géographique, utilisé pour servir de base à la mise en pratique décentralisée des politiques nationales. Il n'est pas rare que le nom d'un Département fasse référence à des éléments relatifs à la géographie physique avoisinante : fleuve ou rivière (Tarn, Cher, Rhône, ...), massif montagneux et/ou littoral (Pyrénées Orientales, Hautes Alpes, ...). Supprimer les Conseils Généraux ne va pas faire disparaître ces éléments naturels ni opérer une modification des cartes de géographie de la France enseignées

\footnotetext{
${ }^{7}$ Éventuelle, voire simplement de leur changement de statut, comme nous le verrons par la suite.

${ }^{8}$ Alors même en 2006 qu'il ne s'agissait même pas d'un projet mis à l'agenda politique à court ou moyen terme, contrairement à l'annonce faite en 2014 par Manuel Vals, 88\% des français manifestaient déjà spontanément leur hostilité à la suppression éventuelle de leur département (Pasquier 2007).
} 
à l'école. Il demeurera tout à fait possible que les habitants de la Vendée revendiquent leur fibre vendéenne même si leur Département n'a plus le statut de Collectivité Territoriale. Cela n'empêche pas non plus les habitants du sud des Pyrénées Atlantiques de se sentir basques même si aucun Département basque n'existe dans l'hexagone, à l'image du sentiment développé vis à vis des « pays ».

On perçoit bien à quel point la confusion terminologique entre Départements et Conseils Généraux s'avère stratégique et providentielle pour la survie de ces derniers. Le Lidec (2007) rappelle d'ailleurs que c'est bien suite aux conseils prodigués en 1999 par une agence de communication que l'assemblée des Présidents de Conseils Généraux a choisi de se rebaptiser Assemblée des Départements de France (ADF). Rien de tel, en effet, que de relier son destin à celui d'une circonscription administrative et électorale qui, pour sa part, n'est remise en question par personne, et par la même occasion de bénéficier du soutien implicite de l'ensemble des administrations de l'Etat organisées sur une base départementale.

\section{2 : La supposée disparition des dépenses sociales engagées par les Conseils}

\section{Généraux}

1.2.1 : Entre permanence des missions accomplies par l'échelon départemental et perte du statut de Collectivité Territoriale

«Une question (...) pertinente (...est) de se demander si le Département en tant que Collectivité Territoriale ne devrait pas être supprimé » (Masson 2007 p. 109). Chaque mot importe. Il n'est donc pas question (ce qui n'aurait pas de sens comme nous venons de le rappeler) de suppression des Départements. Il ne s'agit pas forcément non plus de parler de 
suppression des Conseils Généraux. Ce qui est en jeu, c'est la suppression du statut de Collectivité Territoriale dont bénéficie le niveau départemental d'administration territoriale.

L'opinion publique est, depuis l'annonce gouvernementale d'avril 2014, destinataire de messages et prophéties alarmistes, prédisant l'effondrement du système de solidarité organisé jusqu'à présent à l'échelon départemental et notamment la disparition implicite des prestations suivantes : $\mathrm{RSA}^{9}, \mathrm{APA}^{10}, \mathrm{PCH}^{11}$, gratuité des transports scolaires, aides aux investissements pour les Communes, etc. Ainsi, Pierre Izard, Président du Conseil Général de Haute Garonne, déclarait-il ${ }^{12}$ : «Les agents (du Conseil Général) qui sont les porteurs des valeurs de proximité et de solidarité (...) seraient donc à terme condamnés à disparaître (...). Il s'agit, avec gravité, de pointer du doigt qu'en supprimant le Conseil Général on aggraverait les inégalités sociales et territoriales, on détruirait les dispositifs de solidarité et de proximité (...), on priverait (les citoyens) des plus élémentaires politiques publiques d'égalité et de solidarité ». A l'appui de son cri d'alarme, il dénonce un manque de dialogue et de concertation du gouvernement ${ }^{13}$ «jamais vu dans notre système démocratique ».

S'il prend le soin de préciser «qu'il ne s'agit pas (pour lui) ici de plaider en faveur d'un niveau de Collectivité », et donc indirectement en faveur du maintien des mandats électifs des conseillers généraux français, le fait de prétendre de façon totalement fallacieuse que la suppression des Conseils Généraux entraînerait la suppression des missions assurées par ces structures (ainsi que celles des fonctionnaires assurant ces missions), laisse croire plutôt le contraire.

\footnotetext{
${ }^{9}$ Revenu de Solidarité Active

${ }^{10}$ Allocation Personnalisée d'Autonomie

${ }^{11}$ Prestation de Compensation pour le Handicap

${ }^{12}$ Propos retranscrits dans la presse quotidienne régionale, notamment La Dépêche du Midi, jeudi 10 avril, p.22.

${ }^{13}$ En dépit du fait qu'il possède la même étiquette politique
} 


\subsection{2 : Suppression des Conseils Généraux: une mesure génératrice d'économies ou de} surcô̂ts?

Le but ne serait bien évidemment pas de supprimer la politique sociale, mais avant tout, dans un premier temps, de faire une croix sur certains frais de structure (indemnités des élus, personnels des groupes, bureaux). L'empilement des niveaux «ne serait qu'anecdotique s'il n'entraînait des coûts de fonctionnement prohibitifs et un invraisemblable gaspillage des deniers publics »(Masson 2007 p.109). Seraient également supprimées des rivalités et doublons entre Collectivités sur des compétences identiques, les circuits administratifs s'en trouveraient simplifiés et la cohérence des politiques locales améliorée (précisément grâce à la fin des rivalités entre Collectivités compétentes sur un même territoire).

Ainsi, des économies plus substantielles passeraient ensuite par l'activation d'économies d'échelle en réaffectant certaines compétences départementales aux Intercommunalités ou aux Régions (à l'image notamment de la gestion des collèges, de celle des routes, ou encore du transport en autobus pour améliorer les correspondances avec les $\mathrm{TER}^{14}$ ). C'est d'ailleurs le scénario privilégié par le CESE pour lequel des Intercommunalités dotées d'un périmètre rationnel «pourraient se voir confier (les tâches) actuellement exercées par le Département en matière sociale ${ }^{15}$ afin (...) d'améliorer l'efficacité des actions » (Roulleau 2009 p. 27).

Notons à ce sujet que les lobbies en faveur des Départements défendent au contraire l'idée qu'une suppression de l'échelon départemental coûterait davantage qu'elle n'engendrerait d'économies, usant de la tribune sénatoriale pour parler d'un «surcoût

\footnotetext{
${ }^{14}$ Trains Express Régionaux

${ }^{15}$ Soit quasiment deux tiers de l'activité des Conseils Généraux mobilisant $80 \%$ de leur personnel (Doré $2007 \mathrm{p}$. 94 ; Portal 2012).
} 
éventuel $^{16}{ }$ lié à l'empilement des niveaux et les doublons et redondances sous-jacents qui n'aurait «fait l'objet d'aucune évaluation précise »(Krattinger \& Gourault 2009a p.71).

A l'inverse, selon une étude ${ }^{17}$ financée par l'Assemblée des Départements de France (ADF), les gains résultant d'une disparition des Départements (fusionnés dans les Régions) «seraient peu significatifs à long terme ${ }^{18} »$ (ibidem). Si c'est une preuve mathématique intangible d'exigent les conseillers généraux et sénateurs, il semblerait qu'eux mêmes en soient également dépourvus.

\subsection{3 : Les actions des lobbies départementalistes}

Mais au delà des nuances terminologiques en jeu ci-dessus, pourquoi ôter le statut de Collectivité Territoriale à l'échelon départemental signerait-il la fin des missions remplies par ces structures ? Le fait que les personnes âgées soient encouragées à vieillir chez elles dépend du budget consacré aux aides allouées à ces personnes et non pas du statut juridique de l'organisation qui redistribue cet argent. De nombreux services et administrations (Éducation Nationale, Santé, Pôle Emploi...) fonctionnent à partir d'agences déconcentrées sans disposer du statut de Collectivité et sans que cela n'entrave en rien la mise en œuvre de leur mission. Si les «Départements » disparaissaient, les SDIS (Services Départementaux d'Incendie et de Secours, exemple parmi tant d'autres), ne cesseraient pas de fonctionner pour autant !

\footnotetext{
${ }^{16}$ Souligné par nous.

${ }^{17}$ Note relative aux enjeux d'une éventuelle fusion Départements Régions, KPMG, publiée par Les Échos le 6 février 2009.

${ }^{18}$ Le CESE partage ce point de vue quand il souligne que la suppression des départements « entraînerait des réorganisations coûteuses sans certitude quant au gain d'efficacité » (Roulleau 2009 p. 22).
} 
C'est donc bien leurs propres intérêts et non pas ceux des administrés que les lobbies d'élus locaux tentent de sauvegarder. L'activité du comité Balladur en apporta une preuve supplémentaire. Il est intéressant de relever que toutes les propositions formulées par ce même comité ont recueilli l'unanimité des membres le composant, à l'exception de quatre d'entre elles (Verpeaux 2009). Or ces quatre propositions ont un point en commun : la volonté de sauvegarder les Départements.

On retrouve en effet cette dimension dans les propositions $n^{\circ} 3$ (suppression des cantons), $\mathrm{n}^{\circ} 8$ et 18 (création de 11 Métropoles ainsi que du Grand Paris et donc transfert de compétences départementales vers ces Métropoles), $\mathrm{n}^{\circ} 11$ (retirer la clause de compétence générale aux Départements)

Toutes les autres propositions, même celles qui demeurent aujourd'hui sources de débats animés et/ou celles qui auraient pu permettre aux membres de l'opposition de l'époque de se singulariser, ont généré un accord unanime au delà des opinions partisanes. Citons, parmi les points les plus polémiques, la réduction du nombre de Régions à quinze $\left(\mathrm{n}^{\circ} 1\right)$, l'achèvement de la carte de l'Intercommunalité $\left(n^{\circ} 4\right)$, le gel des Pays $\left(n^{\circ} 6\right)$, le suffrage universel direct dans les Intercommunalités $\left(n^{\circ} 7\right)$, la création de Communes nouvelles $\left(n^{\circ} 9\right)$, la révision des bases des impôts directs locaux $\left(\mathrm{n}^{\circ} 15\right)$, et enfin le remplacement de la taxe professionnelle par une taxation sur la valeur ajoutée $\left(\mathrm{n}^{\circ} 16\right)$.

Les préconisations des membres du Comité ayant voté contre les quatre propositions précitées confirment sans ambiguïté leur motivation pro Départements. En effet, elles requièrent notamment un élargissement des compétences affectées aux Départements (retirer 
aux Régions la gestion des lycées pour la leur transférer ${ }^{19}$ ), en dépit d'un contexte fréquemment mis en exergue, d'effet de ciseau fragilisant les budgets des Départements et l'exercice réel de leurs compétences actuelles, et tout en ayant voté à l'unanimité la proposition $n^{\circ} 14$ du Comité (le contingentement des dépenses publiques locales).

\section{3 : Les Départements : des territoires de proximité à la légitimité historique}

Le Département devrait-il perdurer car il constitue un échelon de proximité, permettant une accessibilité préservée des populations à tout un ensemble de services publics locaux et de prestations sociales? C'est ce que pensent ses adeptes qui vantent son enracinement, sa stabilité, et qui lui attribuent une «légitimité historique » (Krattinger \& Gourault 2009a p.13).

En quelque sorte, l'opportunité de la survie des Départements émanerait de leur ancienneté. En d'autres termes, ce qui était adapté hier l'est forcément aujourd'hui puisque cela a déjà fait ses preuves par le passé. Pourtant, ces mêmes auteurs adoptent un point de vue diamétralement opposé dès qu'il s'agit des Régions, en évoquant leur redécoupage en vue d'accroître leur puissance, leur visibilité, leur influence, et surtout afin «d'assurer une adéquation avec les territoires vécus que sont les aires d'influence des grandes métropoles régionales » (ibid. p.69).

Laissons de côté pour l'instant cette discussion sur inertie versus innovation organisationnelle et revenons plutôt sur les notions de proximité et d'accessibilité, comme remparts face à une France centralisée et jacobine, en rappelant que les Départements ont été

\footnotetext{
${ }^{19}$ Proposition formulée par Pierre Mauroy et André Vallini.
} 
construits, à l'origine, de façon à ce que leur chef lieu soit accessible en une journée de trajet à cheval au maximum depuis chacun de ses points. Sans pervertir l'esprit qui a animé les créateurs des Départements lors du XVIII ${ }^{\circ}$ siècle, on pourrait alors presque aujourd'hui envisager, via le transport routier ou ferroviaire, la Région ${ }^{20}$ comme héritière de cet esprit de proximité de l'administration territoriale. Un chef lieu de Région est en effet beaucoup plus facilement accessible en 2014 que ne l'était un chef lieu de Département en 1790. « Les Régions sont suffisamment proches du terrain pour assumer dans des conditions au moins aussi bonnes l'essentiel des missions des Départements » (Masson 2007 p. 109). Alors même qu'un grand nombre de procédures administratives deviennent en outre dématérialisées et que l'usage d'internet se démocratise, on peut difficilement accuser la suppression du niveau départemental de couper les administrés des services et prestations auxquels ils ont droit.

Les Départements et leurs 4039 cantons ne constituent plus un zonage qui retranscrit la répartition effective de la population et un accès égalitaire aux services publics locaux. Notons d'ailleurs que Roncayolo ${ }^{21}$ fait état de l'existence, dès 1889 , d'une rhétorique visant à condamner les Départements comme des structures inadaptées car ne correspondant (déjà) plus aux territoires vécus. Les écarts de population se sont ainsi très fortement accrus aussi bien entre Départements ${ }^{22}$ qu'entre cantons ruraux en voie de désertification et cantons urbains inclus dans le périmètre d'agglomérations à forte croissance ${ }^{23}$. S'il fallait réformer la carte cantonale pour tenir compte des évolutions démographiques, on aboutirait alors à de

\footnotetext{
${ }^{20}$ Voire des Régions redimensionnées, telles qu'elles pourraient émerger à l'issue du processus de refonte des découpages des Régions également remis à l'agenda au printemps 2014 par le gouvernement Vals.

${ }^{21}$ Dans « Lieux de Mémoire », cité par Estèbe (2007).

${ }^{22}$ Citons par exemple le Nord qui rassemble plus de 2.600 .000 habitants contre seulement 81.000 en Lozère, soit un rapport de 1 à 32 (DGCL 2013).

${ }^{23}$ Ainsi tandis que l'on note des différences sensibles entre les différents cantons composant la Commune de Toulouse (21.000 habitants dans le sixième canton et presque 70.000 dans le quinzième), les écarts sont encore plus caricaturaux vis-à-vis des parties rurales du département (3.700 habitants dans le canton de Saint Béat), voire des parties rurales de départements ruraux comme l'Ariège (532 habitants dans le canton de Quérigut) ou les Hautes Alpes (388 habitants dans le canton de Barcillonnette).
} 
minuscules cantons dans les grandes villes et à des cantons extrêmement vastes en zones rurales et montagneuses.

Les cantons ont donc perdu la pertinence qui était la leur il y a plus de deux siècles, surtout en zone urbaine, pour ne plus être que de simples circonscriptions électorales. « La troupe des conseillers généraux apparaît de plus en plus (...) comme une sorte de (...) vestige du passé de l'institution qui la tire vers le bas et empêche de mettre en œuvre l'intérêt départemental »(Estèbe 2007 p. 122) «La géographie administrative et politique de notre pays ne correspond plus aux données de la vie concrète (...) Des circonscriptions périmées sont maintenues artificiellement. A l'inverse, on prive d'identité juridique des espaces en formation » comme ceux des Intercommunalités (Piron 2006).

Si le zonage cantonal est devenu aujourd'hui totalement inadapté aux zones qui se sont fortement urbanisées, c'est bien le niveau intercommunal qui paraît le plus pertinent pour la mise en oeuvre des politiques de proximité (notamment sociales) auprès des populations. «L'intercommunalité est maintenant apte à gérer l'ensemble des compétences (...) de proximité du Conseil Général»(Guillaume 2010 p. 15). Les Établissements Publics de Coopération Intercommunale (EPCI) exercent d'ailleurs déjà un nombre croissant de compétences à la place, pour le compte, ou à côté des Départements. On peut presque voir en eux les successeurs des cantons ${ }^{24}$ dans la mesure où le périmètre de bon nombre des groupements créés suite à la loi Chevènement ${ }^{25}$ a épousé autant que possible celui du ou des cantons qui préexistaient sur leur territoire, précisément pour anticiper une éventuelle disparition des mandats électifs départementaux.

\footnotetext{
${ }^{24}$ Ils regroupent en moyenne treize Communes là où les cantons en agrègent moins de dix.

${ }^{25}$ Loi 99-586 du 12 juillet 1999 relative au renforcement et à la simplification de la coopération intercommunale.
} 
Nous venons d'envisager trois «mauvaises » raisons de justifier le statu quo territorial et de préserver les Départements français dans notre architecture locale. Appréhendons à présent quatre arguments plus positifs permettant de justifier la suppression de cet échelon départemental non pas pour ce que l'on n'aurait pas à perdre, mais pour ce que l'on gagnerait en l'envisageant.

\section{2 : Pour quelles raisons devrions-nous « supprimer les Départements »?}

Quatre raisons fondamentales d'allègement du millefeuille territorial français, par abandon de son niveau départemental, seront détaillées dans cette seconde partie : libérer les Communes d'une certaine tutelle départementale, se séparer d'un zonage incompatible avec la mise en place d'une dynamique de projets, mettre en accord le statut de l'échelon départemental avec ses prérogatives effectives, et enfin garantir le financement des compétences obligatoires du bloc départemental.

2.1 : Redonner aux Communes une vraie autonomie exempte de toute tutelle départementale

On loue souvent les mérites des Départements en tant que financeurs et soutiens indispensables au financement de l'investissement des Communes (notamment les plus rurales et petites d'entre elles). Cette réalité constituerait une raison fondamentale de ne pas supprimer l'échelon départemental. Pour avoir de la croissance au niveau national, il faut investir, et ce sont essentiellement les Collectivités Territoriales (et surtout les Communes, grâce aux subventions départementales) qui se chargent de l'investissement public en 
France $^{26}$. Supprimer les Départements équivaudrait dès lors à créer des tensions budgétaires insoutenables pour les Communes, qui sacrifieraient dès lors leur investissement, et par voie de conséquence la croissance de la richesse nationale.

Dans cette perspective, les conséquences de la proposition de loi présentée par Jean François Mancel (Assemblée Nationale 2010) visant à interdire les subventions des Départements (et des Régions) aux Communes pourraient paraître funestes. Toutefois, la logique de cette proposition ne fut évidemment pas de paupériser les Communes mais au contraire de plaider pour une fin de la tutelle officieuse qu'exercent les Départements sur les Communes françaises, pourtant prohibée par la Constitution ${ }^{27}$. Supprimer les Départements, loin de pénaliser les Communes, rendrait à ces dernières une autonomie réelle d'action.

Financièrement il est en effet neutre, pour elles, de recevoir directement des concours aux investissements en provenance de l'Etat ou de les obtenir via un intermédiaire (le Département) qui se sert de ce rôle d'intermédiaire pour justifier sa propre existence et contrôler, de fait, leur activité. Le risque de tarissement de cette ressource peut néanmoins sembler plus grand, dans une période où les dotations étatiques ont tendance à se raréfier. Cependant dans la mesure où une part importante des ressources départementales est, elle aussi, dépendante de l'Etat, ce risque pèse peu au final face au gain d'autonomie et d'indépendance politique que les Communes en retireraient.

\footnotetext{
${ }^{26}$ Elles en assurent en effet $71.5 \%$. Au sein des collectivités, c'est le bloc communal (Communes et Intercommunalités, même si ces dernières ne disposent pas du statut) qui supporte quasiment $76 \%$ des dépenses d'équipement contre 17\% pour les Départements et $7 \%$ pour les Régions.

${ }^{27}$ Dans son article 72 alinéa 5.
} 
La suppression des Départements entraînerait donc la disparition de la tutelle implicite qu'ils exerçaient sur les Communes via la détention d'un droit de veto sur le financement de leurs investissements. Ainsi prendraient fin les logiques de discriminations partisanes où, par exemple, un Conseil Général pourrait s’investir de la sauvegarde du patrimoine religieux représenté par les églises présentes sur son territoire, mais ne subventionner la réfection que de celles des Communes dont les maires sont ses alliés politiques.

Outre cet intérêt pour les Communes, la suppression de ces liens financiers transitifs entre Etat, Départements et Communes contribuerait à une plus grande lisibilité démocratique de l'action de chaque niveau de Collectivité (Pissaloux 2009). En parvenant à «mettre fin à la dérive des financements croisés $»^{28}$ (Warsmann 2008 p.69), les citoyens seraient plus aptes à cerner «qui fait quoi », et à démêler les stratégies d'imputation menées par les élus pour s'attribuer le mérite de telle décision porteuse ou au contraire se désolidariser de telle autre décision plus polémique. Les politiques publiques pourraient plus facilement être évaluées.

\section{2 : Supprimer un zonage qui est l'antithèse d'un territoire de projet}

Il est courant de vilipender les excès de centralisation de l'administration française et sa tendance quasi immanente au jacobinisme. Les administrés y voient souvent des démarches complexes et procédurières. Les élus locaux stigmatisent quant à eux davantage l'insuffisance de liberté dont jouissent leurs Collectivités, seules capables de soutenir l'investissement public et de stimuler le développement économique par des dynamiques de projets, et cela sans céder aux dérapages financiers si coutumiers de l'Etat central.

\footnotetext{
${ }^{28}$ Même si l'Etat a pris l'habitude d'en générer d'autres lui même, comme pour le financement des grandes infrastructures qui lui incombe.
} 
La décentralisation au travers de ses différents «actes»s'est, depuis une trentaine d'années, chargée de modifier cette tendance, par différents moyens: transferts de compétences, contractualisation avec les Régions, garantie constitutionnelle de l'autonomie des Collectivités, et plus récemment développement de l'Intercommunalité. Le fil rouge que l'on retrouve dans la plupart de ces actions décentralisatrices peut s'analyser comme une philosophie commune : «appréhender l'Etat comme un problème et les collectivités locales comme une solution » (Bouvier 2010a p. 44). On estime que c'est au niveau local, dans des territoires qui font sens pour leurs occupants, qu'émergera une dynamique de projets, et non pas par le biais de décisions prises d'en haut par l'Etat, déconnecté des réalités et spécificités locales (Houllier Guibert 2010).

L'Intercommunalité promue par la loi du 12 juillet 1999 s'inscrit totalement dans cette démarche puisqu'elle est présentée avant tout comme une Intercommunalité de projets. Une grande liberté est laissée aux élus locaux pour façonner des périmètres à leur convenance, quitte, de façon paradoxale, à ce que ces derniers soient notoirement trop étriqués pour précisément générer de réelles dynamiques de projets et des mutualisations de moyens suffisantes (Thomas 2008). Le législateur, pour convertir les plus réfractaires des élus communaux à l'Intercommunalité de projet, a décidé de leur faire confiance sur la définition des périmètres.

Si un consensus émerge pour affirmer qu'un zonage défini par les acteurs eux mêmes est indispensable à l'activation d'une réelle dynamiques de projets, et si l'on se fixe pour but de façonner progressivement une France de plus en plus décentralisée, notamment grâce aux EPCI, alors la suppression des Départements relèverait davantage de l'aubaine que de la régression. 
En effet, les Conseils Généraux ont compris l'intérêt, pour leur survie en tant que Collectivité Territoriale, de freiner l'essor de ces Groupements intercommunaux à Fiscalité Propre (GFP). Car l'essor des uns entraînerait forcément l'affaiblissement des autres. Ils ont d'ailleurs « largement phagocyté l'Intercommunalité la plus susceptible de les menacer » (Le Lidec 2007 p. 115). Ils ont également anticipé l'éventuelle suppression de leur Conseil Général en influençant, via le cumul des mandats, la création de GFP dont les contours se confondent étrangement avec ceux du zonage cantonal. L'impératif d'achèvement et de refonte de la carte intercommunale a dû composer avec cette perversion de la philosophie initiale de la loi, pour stimuler la constitution de GFP dotés de périmètres plus pertinents, donc plus à même, dans un futur proche, de bénéficier de transferts de compétences départementales.

Par ailleurs, gardons en mémoire que les révolutionnaires ont créé les Départements en donnant pour mission à ces derniers non pas de stimuler les particularismes locaux mais au contraire de les gommer au maximum, voire de les éradiquer. Il s'agissait de s'affranchir de toute spécificité issue du passé féodal, d'une France découpée en provinces, de peur que ces dernières manifestent des velléités d'autonomie venant menacer la construction révolutionnaire de la Nation une et indivisible (Présidence de la République 2009). « Ce fut pour détruire toute identité autre que nationale que furent instaurés (...) nos Départements » (Laurent 2008 p.6). Ce n'est pas le fruit du hasard si leurs noms ont été majoritairement empruntés à la sphère géographique, et non pas historique et/ou culturelle. 
Historiquement, le Département fut donc avant tout un outil centralisateur. En tant que zonage d'application des politiques des administrations centrales de l'Etat, il le demeure d'une certaine façon, et le demeurera encore. «Le Département (...) n’est pas un territoire (au sens anthropologique), c'est un périmètre (...) un territoire non vécu et non identitaire, fondé sur l'accessibilité des services, anticipé, pour différentes raisons, dès 1792 » (Estèbe 2007 p. 121). Dans la mesure où le zonage départemental a été imposé aux citoyens et ne répond à aucune logique économique et/ou culturelle (comme les EPCI ou les «pays »), il est logique qu'il ne soit pas le lieu d'émergence de projets et que sa principale utilité soit de faciliter l'application de politiques étatiques au niveau infra national. Cela nous amène à considérer les Conseils Généraux comme une simple catégorie, parmi d'autres, d'agences déconcentrées de l'Etat.

\section{3 : Mettre en accord le statut légal de l'échelon départemental avec ses} prérogatives effectives

\subsection{1 : Les Départements : des Collectivités qui ne maîtrisent plus leurs recettes}

Les Départements français, tels que nous les connaissons aujourd'hui, n'ont en fait de Collectivité Territoriale que le nom. Si l'on s'accorde sur le principe en vertu duquel «il n'y a pas de collectivité locale sans capacité à lever un minimum d'impôts (Comité pour la Réforme des Collectivités Locales 2009 p. 107), alors la façon dont la loi RCT a privé les Départements de la quasi intégralité de leur autonomie fiscale a contribué à affaiblir significativement leur légitimité en tant que Collectivité Territoriale au bénéfice du bloc communal, et notamment des EPCI qui, au contraire, présentent toutes les caractéristiques des Collectivités Territoriales sans en posséder le statut. 
Suite à la RCT, les ressources départementales ont en effet été impactées par les quatre principales évolutions suivantes $^{29}$ : le recentrage autour de la Taxe sur le Foncier Bâti (TFB) du fait de l'adjonction de l'ancienne part régionale de cette dernière, la perte des parts de la Taxe d'Habitation (TH) et de la Taxe sur le Foncier Non Bâti (TFNB), l'affectation d'une partie de l'imposition forfaitaire des entreprises de réseaux (IFER) nouvellement créée, ainsi que l'affectation de $48.5 \%$ du produit de la Cotisation sur la Valeur Ajoutée Economique (CVAE), prévue en remplacement de la Taxe Professionnelle (Floury 2012).

Même si la Constitution est censée garantir aux Départements $58.6 \%$ de ressources «propres ${ }^{30}$, il apparaît que la TFB est le seul élément de ce nouveau paquet de ressources susceptible de conférer au niveau départemental un semblant d'autonomie fiscale (et financière) via la fixation de son taux ${ }^{31}$. Les autres ressources confèrent le même niveau d'indépendance que des dotations et sont, qui plus est pour certaines d'entre elles, génératrices de volatilité et d'insécurité, à l'image de la CVAE et des DMTO ${ }^{32}$, sensibles aux évolutions conjoncturelles (Guengant 2010). Souvent fixé au maximum, le taux de DMTO est certes ajustable, mais essentiellement à la baisse (Standard \& Poor's 2010).

L'écueil majeur pour les Départements serait alors de subir une baisse drastique du pourcentage de leurs ressources sur lesquelles ils conserveraient une marge de manœuvre, que

\footnotetext{
${ }^{29}$ Sans compter la Taxe Spéciale sur les Conventions d'Assurance (TSCA), et sans revenir sur l'importance de la fiscalité sur les droits de mutation.

${ }^{30}$ Nous ne reviendrons pas ici sur les subtilités de langage qui permettent de respecter ce critère en privant certaines collectivités de toute faculté d'agir sur la base et/ou sur le taux des impôts qu'ils perçoivent.

${ }^{31}$ Il s'agit donc d'un retour en arrière patent concernant le point majeur fondateur de l'autonomie des collectivités locales dès 1980 (loi du 10 janvier) avant même les lois de décentralisation de 1982 : le vote des taux des impôts directs locaux par les assemblées délibérantes des collectivités.

${ }^{32}$ Droits de Mutation à Titre Onéreux, qui représentaient 20\% des recettes départementales en 2009.
} 
d'aucuns ${ }^{33}$ estiment à seulement $16 \%$, contre 35\% avant la réforme (Moraud 2011, Portal 2012, Carrez \& Thénault 2010). En élargissant aux Départements l'analyse de Charles de Courson relative aux Régions, on peut s'interroger sur la pertinence du maintien du statut de Collectivité administrée par des membres élus au suffrage universel direct. Si depuis le milieu des années 80 l'Etat a bâti un «édifice fiscal disparate et constamment ravaudé » (Gilbert 2010 p. 28) et s'est substitué au contribuable local en multipliant dégrèvements et exonérations compensés par des dotations (Bouvier 2009, Bouvier 2010b), et si dès lors les Départements ne sont presque plus chargés «que de gérer (ces) dotations, à quoi bon aller voter lors des prochaines élections (cantonales) » (Assemblée Nationale 2009b p. 9) ?

Deux raisons nous incitent cependant à nuancer la force du lien entre Collectivités Territoriales (ici les Départements) et autonomie fiscale. D’une part, certains auteurs $^{34}$ n'hésitent plus à considérer cette notion d'autonomie fiscale locale comme étant «anachronique » (Bouvier 2010a p. 44), et donc tout sauf consubstantielle de la notion de Collectivité Territoriale. L'autonomie financière des Collectivités serait désormais davantage perçue (par l'Etat) comme un problème, et non plus comme une solution, ce qu'elle incarnait il y a une trentaine d'années (Bouvier 2009). D'autre part, de façon plus pragmatique, il semblerait que la vraie autonomie des Collectivités réside davantage dans la liberté d'utiliser à leur guise les ressources dont elles disposent que dans le fait de récupérer elles-mêmes des ressources $^{35}$, si elles sont ensuite contraintes de les affecter à des emplois qu'elles n'ont pas choisis (Laurent 2010, Gilles 2010).

\footnotetext{
${ }^{33}$ Certains, à l'image d'Éric Doligé lors d'une réunion de la commission des Finances du Sénat le 9 septembre 2009 , sont plus pessimistes et avancent la valeur de $12 \%$.

${ }^{34} \mathrm{Au}$ premier rang desquels figure le directeur de la Revue Française de Finances Publiques, Michel Bouvier

35 Notamment en influençant le produit perçu grâce au vote des taux des impôts dont elles disposent.
} 
2.3.2 : Les Départements : des entités dépourvues d'autonomie stratégique car privées de la maîtrise de leurs dépenses

Les Länders allemands, souvent érigés en modèles de Collectivités dynamiques, ne bénéficient de quasiment aucune autonomie relative à leurs ressources (Thoumelou 2011). S'ils parviennent à être des acteurs moteurs pour leur territoire, c'est par le biais de leurs dépenses librement consenties, et donc de la possibilité d'appliquer la politique de leur choix (Arthuis 2009). A l'inverse, le fait que les Départements français soient grandement dépourvus d'une telle liberté les cantonne en quelque sorte au rang de Collectivités Territoriales «factices », bénéficiaires non pas de la décentralisation mais de simples délégations de compétences (Gourault 2011).

L'autonomie politique des Départements se trouve, en effet, de plus en plus contrainte par la hausse de certaines catégories obligatoires de dépenses sur lesquelles ils n'ont que très peu de leviers d'action, à l'image des dépenses sociales qui constituent l'essentiel de leur cœur de métier (Oliva 2012). La crise économique et le vieillissement de la population vont mécaniquement augmenter le volume de prestations devant être versées, sans qu'il soit possible pour les Départements de s'ajuster, par exemple en diminuant les montants alloués ou en réduisant le nombre de bénéficiaires. Ils ne contrôlent en effet ni le niveau de service, ni les conditions d'accès aux prestations qu'ils délivrent (Portal 2012), ayant subi de la part de l'Etat une externalisation du « mauvais risque » $(\text { Le Lidec } 2007)^{36}$.

\footnotetext{
${ }^{36}$ À l'image, en économie de l'entreprise, des donneurs d'ordre qui se recentrent sur leur cœur de métier et qui externalisent auprès de fournisseurs toutes les tâches trop génératrices de coûts fixes, à la rentabilité trop faible, sans toujours se soucier ensuite de l'aptitude de ces fournisseurs à survivre face à ces contraintes (notamment de gestion des stocks) externalisées.
} 
L'autonomie et la libre administration, quoique garanties officiellement la Constitution font, concrètement, cruellement défaut aux Départements. La réforme constitutionnelle de 2003, via l'article 72 de la Constitution, précise pourtant que «les collectivités locales ont vocation à prendre les décisions pour l'ensemble des compétences qui peuvent le mieux être mises en œuvre à leur échelon ». Or l'élargissement des compétences des Départements ${ }^{37}$ ne s'est pas accompagné d'une faculté à prendre des décisions relatives à ces mêmes compétences. Alors que l'Acte II de la Décentralisation ${ }^{38}$ avait été pensé par Jean Pierre Raffarin pour être régionaliste, il a au contraire pris la forme d'un alourdissement des compétences (que d'aucuns qualifieraient plutôt de contraintes) dévolues aux Départements, analysé comme une victoire à la Pyrrhus pour ces derniers : «Ces transferts n'apportent pas aux Conseils Généraux de pouvoirs supplémentaires. Bien au contraire ils tendraient à les réduire (...). Le législateur a très fermement encadré ces secteurs transférés, (...) jusqu'à faire (des Départements) de purs exécutants de la politique nationale » (Estèbe 2007 p. 122), ce qu'ils ont toujours été par nature, puisque «cette dimension d'exécution est inscrite dans (leurs) gènes même » (ibid. p. 123).

Avant d'être le siège d'une Collectivité, n'oublions pas que les Départements ont d'abord été pensés comme des relais du pouvoir central (Marcou 2011). Bien que non officielle et plus insidieuse, la tutelle de l'Etat n'en est pas moins réelle. N'oublions pas, en effet, que les Départements (comme les autres niveaux de Collectivités Territoriales) n'ont pas la compétence de leur compétence.

\footnotetext{
${ }^{37}$ loi du 20 juillet 2001 qui a créé l'APA, loi du 18 décembre 2003 qui a décentralisé la gestion du RMI, loi du 11 février 2005 qui a instauré la $\mathrm{PCH}$, et loi du $1^{\text {er }}$ décembre 2008 qui a généralisé le RSA.

${ }^{38}$ Loi n $^{\circ}$ 2003-1200 du 18 décembre 2003 portant décentralisation en matière de revenu minimum d'insertion et créant un revenu minimum d'activité ; loi n 2004-809 du 13 août 2004 relative aux libertés et responsabilités locales.
} 
Les Conseils Généraux, cantonnés au rôle de simples exécutants d'une politique nationale décidée par l'Etat (comme le sont d'ailleurs bon nombre de directions territoriales en charge de services déconcentrés), seraient devenus en quelque sorte de gros $\operatorname{CCAS}^{39}$, des «services déconcentrés de l'Etat, sans marge de manœuvre (...) réelle » (Krattinger \& Gourault 2009b p. 176), des «structures de gestion et de redistribution de prestations sociales » (Doré 2007 p. 93), ou encore des «établissements publics spécialisés dans les affaires sanitaires et sociales, (eux) qui, trop souvent, (font) simplement ruisseler les crédits de l'Etat vers les Collectivités infra départementales » (Auber 2011 p.10).

La rigidification de leurs dépenses réelles de fonctionnement, qui croissent plus vite que leurs recettes réelles de fonctionnement depuis 2001, prive les Départements des rares marges de manœuvre dont ils disposaient encore ... d'autant plus quand les Présidents de Conseils Généraux, dans leur majorité, ont fait savoir qu'ils refusaient de considérer la lutte contre la fraude et les prestations indûment perçues comme une tâche leur incombant. L'agencification ${ }^{40}$ des Départements (Estèbe 2007) serait donc désormais quasi irréversible, d'autant plus qu'on peut considérer qu'il s'agit d'une transformation déjà à l'œuvre dans les faits depuis des années (Procureur 2010, Procureur 2012, Laurent 2010, Pinson \& Galimberti 2013).

En retirant aux Départements leur statut de Collectivité Territoriale, on ne ferait, en fin de compte, qu'entériner cette situation et ajuster le statut juridique qui sied le mieux aux Conseils Généraux en fonction de l'exercice concret de leurs missions. Et par la même occasion on les mettrait dans un contexte institutionnel plus favorable, car paradoxalement plus contraint, pour précisément assurer la pérennité de l'exercice de ces mêmes missions.

\footnotetext{
${ }^{39}$ Centre Communal d'Action Sociale.

${ }^{40}$ Selon le néologisme de Renaud Epstein, 2013, «La rénovation urbaine. Démolition reconstruction de l'Etat », Paris, Les Presses de Sciences Po, collection « Sciences Po Gouvernances, 377 pages.
} 


\section{4 : L'abandon de la clause générale de compétence : une mesure salutaire pour}

\section{le financement du bloc de compétences départementales}

\subsection{1 : Contrainte budgétaire accrue et remise en cause de l'aptitude à initier des politiques} d'intérêt départemental

Pour garantir leur indépendance politique et, somme toute, pour légitimer leur existence face aux menaces récurrentes de disparition (Hertzog 2012), la tentation est grande pour les Départements de faire usage de leur clause de compétence générale et donc de s'auto saisir du plus grand nombre de problèmes possible, à des fins de constitution de réseaux et d'influences politiques vis à vis de différentes catégories d'électeurs. On estime que $18 \%$ des dépenses des Départements seraient non obligatoires et viendraient donc entamer l'enveloppe financière (de plus en plus contrainte) disponible pour l'exercice des compétences sociales obligatoires déléguées par l'Etat (Portal 2010) ${ }^{41}$.

Certains estiment «simpliste et illusoire » de recommander «aux Départements de se recentrer sur leurs compétences obligatoires »(Jamet 2010 p. 76), arguant de «l'impact social réel » des politiques culturelles et sportives facultatives. Néanmoins la question ne serait pas celle de l'utilité de ces politiques facultatives ressentie par tel ou tel public, mais plutôt celle de l'identité de la Collectivité qui l'entreprend ainsi que celle de la survie financière de cette dernière. Se poseraient sinon, à contrainte budgétaire donnée, des problèmes sans fin d'arbitrage entre différentes politiques publiques dans différents domaines.

\footnotetext{
${ }^{41}$ L'auteur se réfère lui-même à l'étude d'impact sur le projet de loi RCT. Pour sa part, Moraud (2011), se référant à Carrez \& Thénault (2010), estime ces dépenses hors compétences légales davantage entre 10 et 15\%.
} 
En effet, suite aux crises des subprimes et des dettes souveraines ayant entraîné conjointement l'explosion des dépenses sociales obligatoires des Départements et le tarissement du dynamisme de leurs recettes fiscales (perte de plus de 2.5 Md€ de DMTO entre 2008 et 2009) ${ }^{42}$, la situation financière de certains de ces derniers est devenue inextricable. En 2010 plusieurs sources ${ }^{43}$ ont recensé une trentaine de Départements en grande difficulté financière, du fait de ce classique effet de ciseau entre dépenses contra cycliques servant d'amortisseurs et contraction des recettes (Moraud 2011, Carrez \& Thénault 2010). Et projections pour 2015 sont encore plus alarmistes puisque certains scenarii envisagent jusqu'à deux tiers de Départements en difficulté ${ }^{44}$. Les taux de couverture ${ }^{45}$ se sont aggravés sensiblement de 2008 à 2010 (Balcon 2010, Portal 2012), aussi bien pour des prestations notoirement sous financées (de $32.9 \%$ à $28.7 \%$ pour l'APA) que pour d'autres au financement initialement plus équilibré (de $96.8 \%$ à seulement $47.8 \%$ pour la $\mathrm{PCH})^{46}$.

Le fait que dépenses et recettes réelles de fonctionnement croissent de façon déconnectée les unes des autres se traduit automatiquement par une dégradation du taux d'épargne brute ${ }^{47}$ qui a atteint un niveau plancher historiquement bas (à $10.9 \%$ en 2009 contre 18.1\% en 2006), qu'il peine à abandonner aujourd'hui : 11.4\% en 2013 (Observatoire des Finances Locales 2013 p.59). Il en résulte automatiquement un poids plus lourd de la dette des Départements, appréhendé par leur capacité dynamique de désendettement ${ }^{48}$, ce qui peut poser des problèmes insurmontables de soutenabilité budgétaire aux Départements, soumis à

\footnotetext{
${ }^{42}$ Alors que ces DMTO augmentaient avant la crise des subprimes, sur la période 2001-2006, de 12,5\% par an (Doré 2007 p. 91).

${ }^{43}$ On peut également mentionner l'étude de Standard \& Poor's d'avril 2010.

44 Guengant, Alain \& Gilbert, Guy, 2010, «Quels budgets pour les départements à l'horizon 2015? Prospectives financières », ADF, Avignon, 20 octobre 2010.

${ }^{45}$ Différence entre les dépenses engagées et le reste à charge, en pourcentage.

${ }^{46}$ Le RSA présente quant à lui un taux de couverture stable autour de $90 \%$.

${ }^{47}$ Ratio entre l'épargne brute et les recettes de fonctionnement.

48 Avant la crise (2007), la dette des départements représentait l'équivalent de 2.5 années d'épargne brute; pendant et après la crise (2009), cette valeur a plus que doublé (5.2 années).
} 
la règle d' or ${ }^{49}$ qui leur interdit de s'endetter pour rembourser leur dette et les oblige à dégager une épargne brute suffisante pour être solvables (Portal 2012).

Qui plus est, même en dehors de périodes économiquement instables, les DMTO s'avèrent être des ressources bien trop volatiles et inégalement réparties au niveau géographique $^{50}$ pour couvrir des dépenses sociales contra cycliques. Le risque pour les Départements est de se retrouver confrontés « à la nécessité de réduire en valeur absolue les dépenses correspondant aux services publics les moins essentiels » (Arthuis 2009 p. 17) ${ }^{51}$. Même si Jamet (2010) ne cautionne pas ce choix d'un repli sur les compétences obligatoires, il convient de souligner qu'il ne s'agirait donc pas ici d'un choix (en termes de stratégie politique) mais davantage d'une contrainte et d'une nécessité (financière), qui est d'ailleurs prévue par le $\mathrm{CGCT}^{52}$, ce dernier imposant en effet aux Collectivités de financer en priorité leurs compétences obligatoires (Thoumelou 2011). Les Collectivités Territoriales ne peuvent plus se permettre, comme entre 1983 et 2008, de consacrer $40 \%$ de la croissance de leurs dépenses à des domaines étrangers aux compétences qui leur ont été transférées (Gilbert 2011, Carrez \& Thénault 2010).

\footnotetext{
${ }^{49}$ Article L-1612-4 du Code Général des Collectivités Territoriales (CGCT)

${ }^{50}$ Ainsi le regain des recettes de DMTO à partir de 2010 n'a concerné qu'une minorité de départements, essentiellement ceux d'Ile de France, du pourtour méditerranéen et ceux incluant des grandes agglomérations (Moraud 2011).

51 À l'image des problématiques auxquelles est confronté l'Etat dans de nombreux domaines (qui résout traditionnellement les problèmes d'effet de ciseau par une hausse de l'endettement)

${ }^{52}$ Article L 1111-4.
} 


\subsection{2 : Vers un nécessaire recentrage autour des compétences obligatoires des Départements} agencifiés

«Corriger substantiellement l'architecture du pouvoir local décentralisé est devenu impératif pour assurer la soutenabilité des finances (départementales) 》 (Hertzog 2012 p. 122). En effet, de façon paradoxale, contrairement à la menace brandie par certains Présidents de Conseils Généraux alarmistes, le fait d'ôter aux Départements leur statut de Collectivité pourrait permettre, comptablement, de garantir la pérennité de l'octroi des principaux transferts sociaux gérés au niveau départemental. Les Départements «agencifiés » appliqueraient, exactement comme ils le font aujourd'hui, une politique étatique à partir de ressources qui proviendraient alors intégralement du budget de l'Etat. Cela mettrait fin au problème créé par l'insuffisance des moyens transférés par l'Etat au regard des missions à accomplir (Fleury 2012). Ainsi serait consacré, quoique officieusement, l'abandon de leur clause de compétence générale, au profit du recentrage sur un bloc de compétences, dont l'application avait jusqu'à présent été précisément «tuée dans l'œuf par une clause générale devenue le trop fertile terreau de la jungle des interventions locales » (Lefèvre 2011 p. 11), vouées à «l'anarchie éternelle » (ibid. p.13).

En outre, le fait que les Départements se recentrent sur leurs compétences obligatoires donnerait de sérieux arguments aux Collectivités Territoriales ${ }^{53}$ dans l'éternel débat qui les oppose à l'Etat, quand il s'agit pour elles de financer à sa place (et à sa demande bien sûr) une partie de certains investissements structurants d'ampleur, comme une ligne de train à grande vitesse (LGV) par exemple. Car lorsque des Départements choisissent délibérément, par exemple, de subventionner une Commune d'un autre Département ou de financer des tracts

\footnotetext{
${ }^{53}$ Et l'ensemble des collectivités locales de façon plus générale, à l'image des Régions.
} 
anti européens $^{54}$ (Lefèvre 2011 p.13), quand le Val de Marne doublonne les Communes sur les crèches et que la Seine et Marne fait de même avec les Régions sur les lycées (Gingembre 2009), il leur est ensuite plus difficile de rejeter une éventuelle participation de leur part à la construction d'une LGV sous prétexte qu'il s'agit d'une compétence étatique et non départementale. En effet, en arguant de l'exercice de leur clause générale de compétence, pourquoi ne pourraient-ils pas soutenir qu'une LGV représente un investissement plus porteur pour la dynamique économique départementale que de financer un club de football ${ }^{55}$ ?

Une telle évolution institutionnelle reléguant les Conseils Généraux au rang de simples «guichets de la solidarité nationale» délocalisés (Standard \& Poor's 2010 p.9) serait totalement neutre pour les administrés ayant droit (toujours en contact avec la même administration et percevant les mêmes prestations), tandis qu'elle contribuerait à clarifier les actions mises en œuvre par les Collectivités résiduelles (en limitant les financements croisés). On songe notamment à la culture, au sport et au tourisme qui constituent trois domaines de « déploiement de moyens concurrents et redondants sur un même territoire » (Lefèvre 2011 p. 13). Les caisses de Sécurité Sociale gèrent de très nombreux dossiers et sont indispensables à l'ensemble des citoyens sans pour autant que l'on ait besoin d'élire des personnes à leur tête. Les Départements actuels sont en fait dans une situation analogue : «plus besoin d'élus, des administrateurs nommés feront l'affaire »(Laurent 2010 p. 42).

Certains (Doré 2007 p. 96) sous entendent même que ce recentrage des Départements sur leurs missions essentielles, et le quasi abandon, de fait, de l'exercice de leur clause de compétence générale, «était peut être l'objectif implicite des transferts de compétences opérés

\footnotetext{
${ }^{54}$ Se pose également le problème du temps et des moyens consacrés à exercer un contrôle de légalité sur les mesures résultant de l'exercice de la clause de compétence générale des collectivités, épineux sujet que nous ne détaillerons pas dans la présente contribution.
} 
par l'Etat ». Les multiples atermoiements sur cet épineux sujet de la suppression de cette clause pour les Départements accréditent la pertinence de cette hypothèse d'une voie plus insidieuse pour ôter aux Départements l'une des fondements de leur statut de collectivité locale, «comme si, à défaut de leur suppression (...) les dépenses sociales allaient peu à peu les absorber »(Jehel 2007 p. 103).

\section{Conclusion : réformer les Départements ou réformer le Sénat ?}

Une réforme de la Constitution sera, quoi qu'il en soit, nécessaire pour pouvoir se débarrasser des Départements, dans la mesure où ces derniers sont explicitement mentionnés en tant que Collectivités Territoriales dans son article 72. Étant données les forces de résistance qui s'expriment traditionnellement au Sénat à ce sujet, et pour court-circuiter « l'hégémonie des pouvoirs installés » (Guillaume 2010 p. 16), le référendum apparaît être le seul moyen d'action.

Mais au lieu d'interroger les français sur la suppression du niveau départemental de Collectivité Territoriale, on pourrait également s'interroger sur la notion même de bicamérisme et envisager, en amont, la suppression du Sénat. La stratégie qui consiste à ce qu'un Président de Conseil Général (également parlementaire) fasse en sorte d'être désigné rapporteur d'un projet de loi qui pourrait venir éroder l'institution départementale a été maintes fois mise en pratique (Le Lidec 2007). Ainsi, c'est bien au niveau sénatorial que sont bloquées toutes les réformes ambitieuses et que se consolide l'inertie de notre système, du fait du droit de priorité dont dispose le Sénat dans l'examen des projets de loi ayant pour principal objet l'organisation des Collectivités Territoriales ${ }^{56}$.

\footnotetext{
${ }^{56}$ En vertu de l'article 39 de la Constitution suite à la révision du 28 mars 2003.
} 
De façon récurrente, «la préservation des pouvoirs existants a conduit les sénateurs à refuser d'anticiper les conséquences des évolutions en cours » (Guillaume 2010 p. 17) dès que la dimension départementale était susceptible d'être impactée, même indirectement. Les sénateurs oeuvrèrent notamment pour refuser le statut de Collectivité Territoriale de plein exercice initialement prévu pour les Métropoles (créées par la RCT). Le péril était ici évident : se faire évincer par une Collectivité hostile dotée de la clause de compétence générale, et habilitée à récupérer l'ensemble des compétences départementales appliquées sur les Communes la composant (Caillosse 2010).

A défaut de supprimer le Sénat (voie plus périlleuse encore que la voie référendaire pour supprimer uniquement les Départements), on peut aussi choisir des voies plus détournées et plus lentes, comme la division par deux du nombre d'élus envisagée par la RCT via l'instauration des conseillers territoriaux, mais abrogée avant d'être appliquée ... ou encore la promotion de l'Intercommunalité en espérant qu'elle marginalise à elle seule le niveau départemental ... ou enfin, à un niveau moindre, l'interdiction du cumul des mandats d'élu local et de parlementaires (Jégouzo 2010) qui touche $80 \%$ de ces derniers.

Mais finalement on peut aussi penser qu'agencifier le Département de façon croissante, l'asphyxier financièrement pour mieux contrôler ses dérives interventionnistes, sans pour autant le priver officiellement de sa clause de compétence générale est la solution la plus efficace et la plus vraisemblable, à défaut d'être rapide et courageuse. Si l'on ne peut imposer la suppression du niveau départemental, autant organiser alors méticuleusement, par étapes, le dépérissement des Conseils Généraux, et opter pour «les vertus du silence et de l'ambiguïté » (Le Lidec 2009 p. 487). Le recours à la ruse de la part de l'Etat est-il la stratégie 
la plus adaptée ${ }^{57}$ ? L'urgence n'est-elle devenue suffisamment forte pour qu'on délaisse des scénarii de ce type et qu'on opte pour une réforme plus radicale?

\section{Bibliographie}

Arthuis, Jean, 2009, «Réforme des Finances Locales et de la Taxe Professionnelle », rapport d'information du Sénat, ${ }^{\circ} 579,21$ juillet 2009, 88 pages.

Assemblée Nationale, 2009a, «Proposition de Loi relative à la Simplification de la Carte des Territoires », présentée par Christian Jacob, n 1401, 23 février 2009.

Assemblée Nationale, 2009b, Compte rendu $\mathrm{n}^{\circ} 120$ de la Commission des Finances, de l’Économie Générale et du Contrôle Budgétaire, 9 septembre 2009.

Assemblée Nationale, 2010, «Proposition de Loi visant à Rendre aux Communes leur pleine Autonomie Financière », présentée par Jean François Mancel, n 3001, 7 décembre 2010.

Auber, Emmanuel, 2011, «La loi RCT ou l'ignorance des territoires», Pouvoirs Locaux, $\mathrm{n}^{\circ}$ 89, pp. 7-12.

Balcon, Jean Bernard, 2010, «Le rapport Jamet sur la situation financière des Départements rappelle à l'Etat ses responsabilités propres, sans rassurer sur l'avenir», Gestion et Finances Publiques, n $^{\circ} 10$, octobre, pp. 715-718.

Balladur, Édouard, 2009, «Pourquoi réformer les Collectivités Territoriales ? », Regards sur l'Actualité, $\mathrm{n}^{\circ}$ 351, pp. 5-8.

Bouvier, Michel, 2009, «La gouvernance financière locale : splendeur, déclin ou renouveau ? », Regards sur l'Actualité, n 351, pp. 39-49.

\footnotetext{
${ }^{57}$ Estèbe (2007) l'envisage tandis que Le Lidec (2007) réfute l'existence même de cette «stratégie de la ruse »
} 
Bouvier, Michel, 2010a, «L'autonomie financière locale: illusion ou refondation? », Pouvoirs Locaux, $\mathrm{n}^{\circ}$ 87, pp. 44-49.

Bouvier, Michel, 2010b, «La réforme de la taxe professionnelle : une refondation de la gouvernance fiscale locale ? », Regards sur l'Actualité, $\mathrm{n}^{\circ}$ 359, pp. 8-23.

Briant (de), Vincent, 2010, «Le fédéralisme administratif : moyen ou fin de la réforme territoriale ? », Pouvoirs Locaux, n 84, pp. 24-29.

Caillosse, Jacques, 2010, «La métropole impossible», Pouvoirs Locaux, n 86, pp. 41-50.

Carrez, Gilles \& Thénault, Michel, 2010, Rapport du groupe de travail de la Conférence sur les Déficits Publics sur «la maîtrise des dépenses locales », mai, 41 pages.

Commission pour la Libération de la Croissance Française, 2008, «Une nouvelle gouvernance au service de la croissance », troisième partie du rapport de la Commission sous la présidence de Jacques Attali, janvier, pp. 178-199.

Comité pour la Réforme des Collectivités Locales, 2009, «Il est temps de décider », rapport présenté au Président de la République par Édouard Balladur, 5 mars 2009, 174 pages.

Direction Générale des Collectivités Locales, 2013, Les budgets primitifs 2013 des Départements, octobre, 39 pages.

Doré, Christophe, 2007, «La situation financière des Départements », Pouvoirs Locaux, $\mathrm{n}^{\circ}$ 75, pp. 91- 96.

Estèbe, Philippe, 2007, « Du conseil général à l'agence départementale ? », Pouvoirs Locaux, $\mathrm{n}^{\circ} 75$, pp. $120-123$.

Fleury, Benoît, 2012, «Le principe de la libre administration des Collectivités Territoriales en matière financière », Gestion et Finances Publiques, n², pp. 150-154. 
Floury, Marie Christine, 2012, «2011 : nouvelle répartition de la fiscalité locale dans les Collectivités Locales », Bulletin d'Informations Statistiques de la DGCL, ${ }^{\circ}$ 85, janvier.

Gilbert, Guy, 2010, «La suppression de la taxe professionnelle : antécédents, enjeux et débats », Regards sur l'Actualité, n 359, pp. 24-38.

Gilbert, Guy, 2011, «Décentralisation et RGPP : la décentralisation et la contrainte financière», Cahiers Français, n 362, pp. 54-59.

Gilles, William, 2010, «Réflexions sur l'autonomie financière des Collectivités Territoriales au regard des réformes de la fiscalité locale», Pouvoirs Locaux, n 87, pp. 109-115.

Gingembre, Etienne, 2009, «Faut-il supprimer les Départements », Capital, n 210, pp. 9294.

Gourault, Jacqueline, 2011, «Une répartition des compétences à clarifier entre Collectivités et avec l'Etat», Regards sur l'Actualité, n 369.

Guengant, Alain, 2010, «Impact de la réforme de la taxe professionnelle sur les perspectives de croissance des budgets locaux», Pouvoirs Locaux, n 87, pp. 55-61.

Guillaume, Yves, 2010, «Pouvoirs locaux et réforme territoriale: changer pour ne pas changer», Pouvoirs Locaux, $\mathrm{n}^{\circ}$ 84, pp. 13-18.

Hertzog, Robert, 2012, «La réforme des Collectivités Territoriales : une ambition financière», Revue Française d'Administration Publique, $\mathrm{n}^{\circ}$ 141, pp. 121-137.

Houllier Guibert, Charles Édouard, 2010, «L'enjeu de la métropolisation en France», Pouvoirs Locaux, $\mathrm{n}^{\circ} 75$, pp. 111-118.

Jamet, Pierre, 2010, «Rapport sur les finances départementales » fait au Premier Ministre, 20 avril 2010, 86 pages. 
Jégouzo, Yves, 2010, «Les chantiers de la simplification », Espaces et Sociétés, $\mathrm{n}^{\circ}$ 142, pp. 117-123.

Jehel, Laurence, 2007, «L'acte II de la décentralisation pour les Départements : une erreur de sémantique ? », Pouvoirs Locaux, n 75, pp. 101-103.

Krattinger, Yves \& Gourault, Jacqueline, 2009a, «Premières Orientations sur la Réorganisation Territoriale », rapport d'information du Sénat, $\mathrm{n}^{\circ}$ 264, 11 mars 2009, 149 pages.

Krattinger, Yves \& Gourault, Jacqueline, 2009b, « Organisation et Évolution des Collectivités Territoriales », rapport d'information du Sénat, n 471, 17 juin 2009, 292 pages.

Laurent, Loeiz, 2008, «Renforcer les Régions et les Intercommunalités en faisant disparaître en 10 ans l'échelon départemental: réflexions sur une proposition du rapport Attali», Pouvoirs Locaux, $\mathrm{n}^{\circ} 78$, pp. 3-7.

Laurent, Philippe, 2010, «Inflation normative, encadrement juridique de la dépense, réduction de l'autonomie fiscale : les libertés réduites par les deux bouts», Pouvoirs Locaux, n 87 , pp. 39-43.

Le Lidec, Patrick, 2007, «Le Département n'est pas insubmersible, même si l'Acte II l'a consolidé», Pouvoirs Locaux, $\mathrm{n}^{\circ}$ 75, pp. 115-119.

Le Lidec, Patrick, 2009, « Réformer sous contraintes d'injonctions contradictoires : l'exemple du Comité Balladur sur la réforme des Collectivités Locales », Revue Française d'Administration Publique, $\mathrm{n}^{\circ}$ 131, pp.477-496.

Lefèvre, Antoine, 2011, «Clarification de la Répartition des Compétences entre l'Etat et les Collectivités Territoriales », rapport d'information du Sénat, $n^{\circ}$ 283, 2 février 2011, 44 pages.

Marcou, Gérard, 2011, «La réforme des Collectivités Territoriales : pourquoi ? », Cahiers Français, ${ }^{\circ} 362$, pp. 30-38. 
Masson, Jean Louis, 2007, «Conseillers généraux : quel mode de scrutin? », Pouvoirs Locaux, $\mathrm{n}^{\circ} 75$, pp. 109-114.

Ministère de l'Intérieur, de l'Outre Mer, des Collectivités Territoriales et de l'Immigration, 2010, «La Réforme des Collectivités Territoriales, une nouvelle impulsion pour les territoires », 12 pages.

Moraud, Jean Christophe, 2011, «La situation financière des Départements français», Revue Française de Finances Publiques, $\mathrm{n}^{\circ} 113$, pp. 257-264.

Négrier, Emmanuel, 2012, «Métropolisation et réforme territoriale», Revue Française d'Administration Publique, $\mathrm{n}^{\circ}$ 141, pp. 73-86.

Observatoire des Finances Locales, 2013, «Les finances des Collectivités Locales en 2013, état des lieux»,9 juillet 2013, 162 pages.

Oliva, Eric, 2012, «Les principes de la libre administration et d'autonomie financière», Revue Française de Finances Publiques, ${ }^{\circ} 119$, pp. 49-69.

Pasquier, Romain, 2007, «L'hypothèse de la rupture», Pouvoirs Locaux, n 75, pp. 124-128.

Pasquier, Romain, 2013, «Gouvernance territoriale : quelles articulations entre Régions et Métropoles ? », Pouvoirs Locaux, n 96, pp. 34-41.

Pinson, Gilles \& Galimberti, Déborah, 2013, « Métropoles franches et Régions agencifiées», Pouvoirs Locaux, $\mathrm{n}^{\circ}$ 96, pp. 48-55.

Piron, Michel, 2006, «Gouverner en France : quel équilibre territorial des pouvoirs ?», Rapport d'information $\mathrm{n}^{\circ} 2881$ au nom de la Commission des Lois, Assemblée Nationale, février. 
Pissaloux, Jean Luc, 2009, «Thème et variations sur la réforme des Collectivités Territoriales, à propos des rapports Lambert, Warsmann, Balladur et Bélot», Gestion et Finances Publiques, $\mathrm{n}^{\circ} 11$, novembre, pp. 812-822.

Portal, Éric, 2010, «Quelles conséquences de l'amoindrissement de l'autonomie financière et

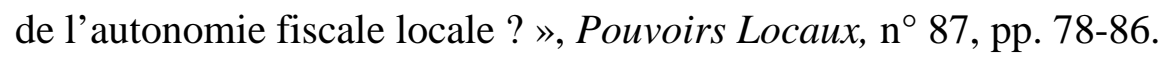

Portal, Eric, 2012, «Quelle soutenabilité des budgets départementaux au regard du poids des compétences sociales, en particulier en matière de vieillissement? », Revue Française de Finances Publiques, ${ }^{\circ}$ 120, pp. 203-218.

Présidence de la République, 2009, Discours du Président de la République sur la Réforme des Collectivités Territoriales, Saint Dizier, 20 octobre 2009.

Procureur, Thomas, 2010, «Le conseiller territorial, figure de proue d'un bateau qui tangue», Pouvoirs Locaux, $\mathrm{n}^{\circ}$ 86, pp. 3-6.

Procureur, Thomas, 2012, «Ce que les conseillers généraux attendent d'un acte III de la décentralisation», Pouvoirs Locaux, n 94, pp. 8-11.

Roulleau, Claude, 2009, Rapport du Comité pour la Réforme des Collectivités Locales, Conseil Économique Social et Environnemental, 10 novembre 2009, 90 pages.

Sadran, Pierre, 2009, «La répartition des compétences entre les Collectivités : quelle clarification?», Regards sur l'Actualité, $\mathrm{n}^{\circ}$ 351, pp. 25-38.

Standard \& Poor's, 2010, «Les Départements français : un système de financement à réinventer », 9 pages, publié le 19 avril 2010.

Thomas, Olivier, 2008, "Intercommunalité française et hausse de la pression fiscale : effet collatéral ou stratégie politique délibérée ?", Revue Française d'Administration Publique, $\mathrm{n}^{\circ} 127, \mathrm{pp} .461-474$. 
Thoumelou, Marc, 2011, «Collectivités territoriales, quel avenir?», Les Études de la Documentation Française, $\mathrm{n}^{\circ}$ 5334-35.

Verpeaux, Michel, 2009, «Le rapport Balladur et les nouvelles structures territoriales», Regards sur l'Actualité, $\mathrm{n}^{\circ}$ 351, pp. 9-23.

Warsmann, Jean Luc, 2008, «La clarification des compétences des Collectivités Locales », Rapport d'information $\mathrm{n}^{\circ} 1153$ au nom de la Commission des Lois Constitutionnelles, de la Législation et de l'Administration Générale de la République, 8 octobre 2008, 123 pages. 